\title{
Stick-slip behavior during electrowetting-on-dielectric: polarization and substrate effects
}

\author{
Russell C. Reid ${ }^{1} \cdot$ Marriner H. Merrill ${ }^{2}$. James P. Thomas ${ }^{2}$ (I)
}

Received: 19 March 2020 / Accepted: 24 July 2020 / Published online: 7 September 2020

(c) The Author(s) 2020

\begin{abstract}
A novel triple-line stick-slip behavior, manifested by "sawtooth oscillations" of the contact angle (CA), was observed during sessile droplet advance by electrowetting-on-dielectric (EWOD) for DC voltages and during droplet retreat for AC voltages. The onset of stick-slip occurred on polished substrate surfaces when the applied potential approached the EWOD saturation voltage and at lower voltages on rougher surfaces. Stick-slip was reduced at higher AC frequencies $(>1 \mathrm{kHz})$, not significantly influenced by $\mathrm{pH}$ or voltage polarity and did not occur with AC polarization on substrates with a Parylene coating but no hydrophobic top-layer. The different triple-line pinning behaviors under DC and AC polarization are shown to be consistent with heterogeneous wetting associated with immobilization of charged species-referred to as charge trapping - near the triple-line at saturation. These experiments and insights offer a new approach for understanding and addressing EWOD device limitations related to CA saturation and charged species trapping leading to improved performance in micro-/ nanofluidic pumps, digital microfluidic chips, and electret devices.
\end{abstract}

\section{Graphical Abstract}
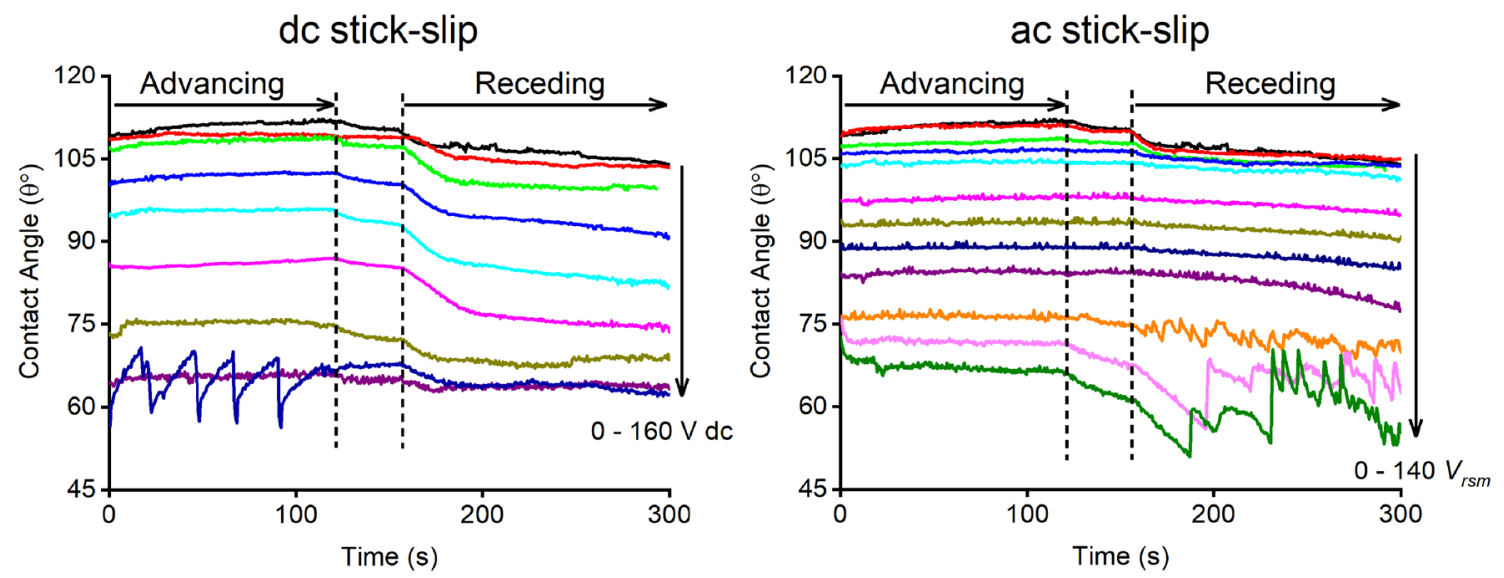

Keywords EWOD · Contact line pinning · Contact angle hysteresis $\cdot$ Charge trapping $\cdot$ Dielectric charging

Electronic supplementary material The online version of this article (https://doi.org/10.1007/s10404-020-02374-y) contains supplementary material, which is available to authorized users.

James P. Thomas

james.p.thomas@nrl.navy.mil

1 Department of Engineering, Dixie State University, St. George, UT, USA

2 U.S. Naval Research Laboratory, 4555 Overlook Ave., SW, Washington, DC, USA

\section{Introduction}

Electrowetting-on-dielectric (EWOD) has emerged as a popular tool for controlling the motion of small liquid volumes because of the low power requirements, relatively fast response with no moving solid parts, and controlled precision. The basic concept involves applying a potential between an electrically conductive liquid and an 
insulated electrode surface, which drives charged species to the solid-liquid interface creating an electric field near the solid-liquid-gas contact line. The resultant force acts to expand the liquid-solid interface producing an apparent decrease in the contact angle (CA) (Mugele and Baret 2005). Lab-on-a-chip devices have been using EWOD digital microfluidics for more than a decade to manipulate individual droplets (Fair 2007; Samiei et al. 2016) and to control liquid in channels or pores (Prins et al. 2001). EWOD is also used in other applications: liquid lenses (Mishra et al. 2014, 2016), screen displays (Charipar et al. 2015; You and Steckl 2010), power conversion from electric to hydraulic (Kedzierski et al. 2016) and mechanical to electric (Krupenkin and Taylor 2011; Wijewardhana et al. 2017), liquid-metal antennas (Diebold et al. 2017), and active surface roughness control (Merrill et al. 2014; Reid et al. 2017).

This paper reports on novel triple-line stick-slip behavior that manifests by "sawtooth oscillations" of the CA of sessile droplets during advance with increasing liquid volume under DC polarization (but not retreat), and during retreat with decreasing volume under AC polarization (but not advance). This behavior only occurred at voltages close to the EWOD saturation potential on ideal surfaces (polished $\mathrm{Si}$ and glass-ITO substrates) and at lower voltages on nonideal surfaces (polyimide layers) (Merrill et al. 2018). The experimental technique used in this work follows surface wetting studies that use an increase or decrease in the droplet volume to drive contact line advance or retreat, but in this case, with an applied potential (constant DC or AC). This technique will be denoted by CP-EWOD for "constant potential EWOD" (Merrill et al. 2018). The more common procedure for EWOD experimentation, potential raster with constant droplet volume, does not show contact line stick-slip in advance or retreat.

Numerous wetting studies (non-EWOD) have addressed contact line stick-slip associated with chemical heterogeneities or rough surfaces that produced CA oscillations during advance and/or retreat and CA hysteresis. Energy models have linked CA hysteresis with metastable states of localized surface energy barriers (Johnson and Dettre 1964; Li et al. 1992; Long et al. 2005). Experiments and modeling have been employed to address stick-slip on surfaces with various wetting heterogeneities on Wilhelmy plate (David and Neumann 2010; Priest et al. 2007, 2013; Shanahan 1991) and sessile drop configurations (Kwok et al. 1998; Leopoldes and Bucknall 2005; Li et al. 2016; Raj et al. 2012; Tavana et al. 2006; Zuo et al. 2012), and surface roughness features with certain characteristics can produce apparent stick-slip (David and Neumann 2013; Huh and Mason 1977; Oliver et al. 1980).

Other authors (Li and Mugele 2008) used a sessile drop CP-EWOD technique to study the effects of polarization magnitude and type (DC/AC) on hysteresis. Their data did not show the stick-slip behavior observed in this work (Fig. 2 in Li and Mugele 2008); however, their maximum applied voltages $\left(160 \mathrm{~V} \mathrm{DC} / V_{\text {rms }}\right)$ in testing appeared to be well below saturation levels for their EWOD system. Another study (Nelson et al. 2011) used a parallel-plate (horizontal) EWOD experiment to generate advancing and receding CAs at the liquid-bridge/plate interface via relative plate motion. Their results (Fig. 7 in Nelson et al. 2011) show stick-slip occurring on both the advancing and receding sides of the liquid-bridge at the highest potential $(60 \mathrm{~V} \mathrm{DC})$ with the slowest plate velocity $(3 \mathrm{~mm} / \mathrm{s})$, but not at lower voltages and/or faster velocities. The CA oscillation was larger on the receding side $\left(\Delta \theta_{r} \lesssim 10^{\circ}\right)$ than on the advance side $\left(\Delta \theta_{a} \lesssim 5^{\circ}\right)$, and the contact line "slip" accelerations were quite large $\left(1000 \mathrm{~m} / \mathrm{s}^{2}\right)$. The authors did not speculate on the mechanism responsible for stick-slip, but they demonstrated that contact line motion fits a spring-mass model with inertial and surface tension forces likely responsible for the oscillating behavior. Other EWOD studies (Gupta et al. 2011; Sen and Kim 2009) reported on stick-slip (quasistatic and dynamic) in the context of hysteresis and pinning in transition between advancing and receding contact line motions. This paper focuses on EWOD stick-slip at saturation, providing experimental data under various conditions and its consistency with possible charge trapping under DC and $\mathrm{AC}$ polarization. The experiments and insights offer a new approach for understanding and addressing EWOD device limitations related to CA saturation and charged species trapping.

\section{Experimental}

In this work, polished glass-ITO and doped silicon substrates were coated with $5 \mu \mathrm{m}( \pm 0.2 \mu \mathrm{m})$ of Parylene-C by vapor deposition. Teflon AF $1600(300 \mathrm{~nm} \pm 50 \mathrm{~nm})$ was applied by spin-coating ( $2 \%$ by weight in Fluorinert FC-40) followed by substrate heating at $70{ }^{\circ} \mathrm{C}$ for $10 \mathrm{~min}$ and then $165{ }^{\circ} \mathrm{C}$ for $10 \mathrm{~min}$. The same spin-coating and heating parameters were used when Cytop $809 \mathrm{M}$ (2\% by weight in CTSOLV180) was used in place of Teflon, producing a coating of similar thickness as the Teflon. The coated substrates had a roughness value of $R_{a}=2-20 \mathrm{~nm}$ as measured with a Tencor P-10 contact profilometer. The EWOD test liquid was a $1: 1(\mathrm{v} / \mathrm{v})$ solution of $0.1-\mathrm{M} \mathrm{NaCl}$ and glycerol, added to minimize evaporation, with a measured surface tension of $65.5 \mathrm{mN} / \mathrm{m}$. A Keithley 2400 SourceMeter was used to apply DC voltages, and an HP 33120A waveform generator with a TREK 2205 high-voltage amplifier were used for applying AC voltages (sinusoidal; 0.5, 1, 2, 5 and $10 \mathrm{kHz}$ ). Voltages were applied between the 30 gauge droplet dispensing needle and grounded substrate (see Fig. 1c). Leakage current under DC polarization was monitored at 

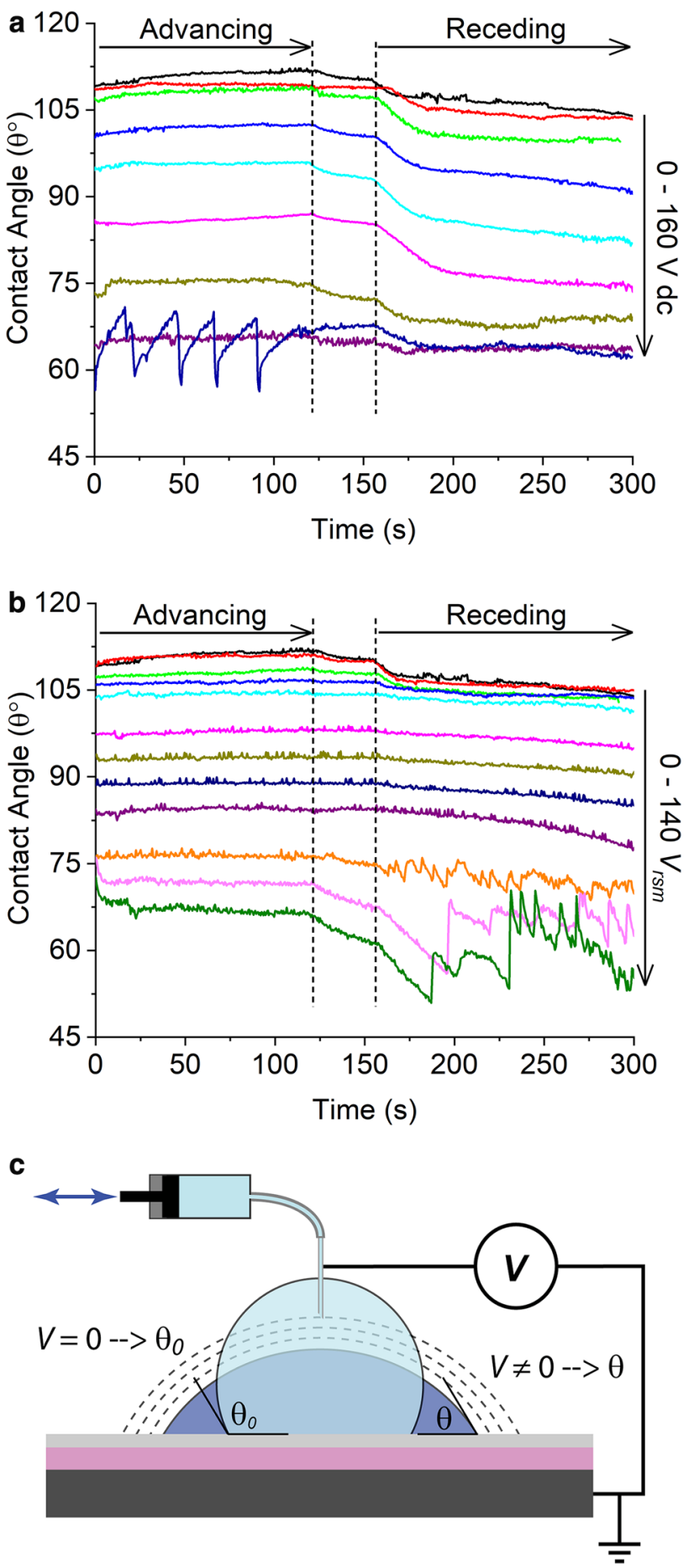

Fig. 1 CP-EWOD plots of advancing and receding CA versus time for DC (a) and AC (b) polarization, and (c) schematic of the CPEWOD technique. In a, the top (black) line is the 0-V DC data, and the potential increases by $20 \mathrm{~V}$ (red) up to $160 \mathrm{~V}$ (blue) for each curve below. In $\mathbf{b}$, the top (black) line is the $0-V_{\text {rsm }} \mathrm{AC}$ data, and the curves correspond to: 13 (red), 25, 38, 51, 64, 76, 89, 102, 115, 127, and 140 (green) $V_{\text {rsm }}$ all times, and the experiment was stopped if it reached or exceeded $1 \mu \mathrm{A}$. Nominal breakdown voltages calculated for the two hydrophobic layers were: Teflon $A F=71 \mathrm{~V}$ and Cytop $=320 \mathrm{~V}$. Droplets $(\sim 5 \mu \mathrm{L})$ were dispensed onto the substrate, a constant DC or AC potential was applied, and liquid was pumped in and then out at $3.5-4 \mu \mathrm{L} / \mathrm{min}$ for 2-3 min in each direction, while measuring the advancing and receding CAs. The needle size and initial (minimum) droplet size were chosen to limit the needle cross-section area to less than $5 \%$ of the droplet's surface area to minimize any possible needle distortion effects on CA measurements (Merrill et al. 2018). CA and droplet diameter measurements were performed using a First Ten Angstrom (FTA) 1000 Drop-Shape Characterization System with FTA32 software analysis of video captures. Additional details are provided in Merrill et al. (2018).

Averaged DC and AC CA responses to EWOD for glass-ITO with Parylene-C and Teflon AF 1600 are shown in Figure S1 in the Online Resource 1 for constant applied voltages ranging from -160 to $160 \mathrm{~V}$ DC and 0 to $144 V_{\text {rsm }}$ AC. ${ }^{1}$ The zero-voltage $\mathrm{CA}$ was $\theta_{0} \sim 108^{\circ}$ and the DC saturation $\mathrm{CA}\left(\theta_{\mathrm{sat}}\right)_{\mathrm{DC}} \sim 63^{\circ}$ occurred at $\left(V_{\mathrm{sat}}\right)_{\mathrm{DC}}>140 \mathrm{~V}$. AC polarization at the DC-equivalent voltage $V_{\text {rsm }} \sim 140$ $\mathrm{V}$ did not show clear signs of saturation. $V_{\text {rsm }}$ was adopted to represent AC EWOD voltage amplitudes instead of $V_{\text {rms }}$ because it provided: (1) a better match between the $\mathrm{AC}$ and DC EWOD CA response data (Figure S1); (2) consistency between observed stick-slip data and predicted changes in $V_{\text {rsm }}$ with charged species trapping; and (3) equivalence between integrated-average charge density at the EWOD solid-liquid interface over a single AC voltage cycle and the charge density associated with a constant $V_{\text {rsm }}$ voltage.

\section{Results and discussion}

Relatively smooth advancing/receding contact lines were observed during EWOD until higher voltages were applied (Fig. 1). DC EWOD (Fig. 1a) showed decreases in the advancing and receding CAs with applied voltage, which were relatively smooth over time for $0-140 \mathrm{~V}$. At $160 \mathrm{~V}$, pronounced stick-slip was observed during contact line advance. Although only $+V$ data are shown in Fig. 1a, $-V$ DC data exhibited similar behavior. AC EWOD (Fig. 1b) showed smooth advancing CA behavior, while the receding contact line began to show signs of stick-slip around

\footnotetext{
${ }^{1} V_{r s m}$, "root-square-mean" voltage, is an alternate representation for an AC voltage in the Lippmann-Young expression. In the absence of trapping: $V_{r s m}=2 A / \pi \sim 0.9 V_{r m s}$, where $A$ is the AC voltage amplitude and $V_{r m s}$ is the standard root-mean-square voltage. Justification and additional information are provided below and in the Online Resource 1.
} 
a dc Polarization

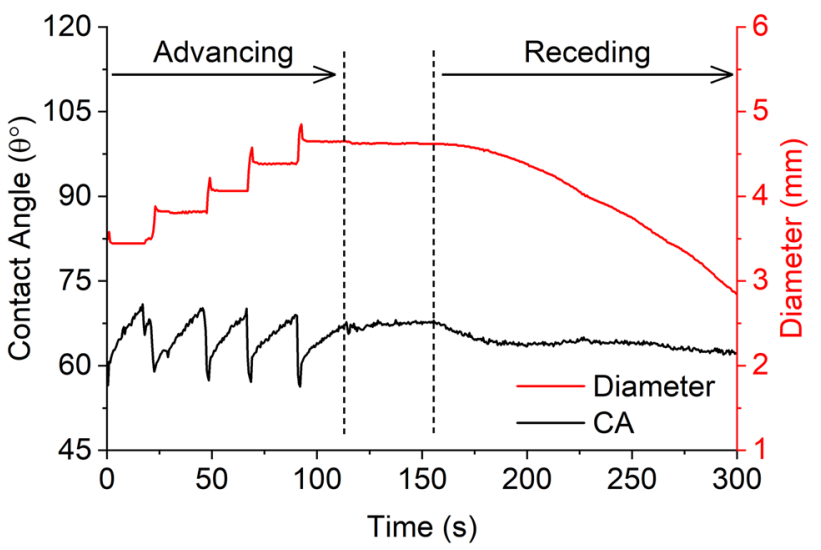

b ac Polarization

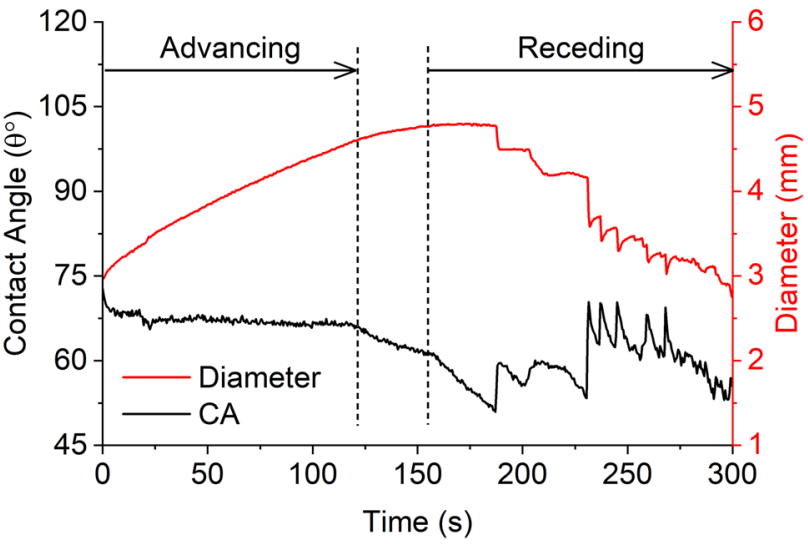

Fig. $2 \mathrm{CA}$ and droplet base width versus time. $V_{\mathrm{dc}}=160 \mathrm{~V}(\mathbf{a})$, $V_{\text {rsm }}=140 \mathrm{~V}(\mathbf{b})$

$V_{\mathrm{rsm}}=115 \mathrm{~V}$ (orange line), and it became more pronounced at higher voltages. CA hysteresis $\left(\theta_{a}-\theta_{r}\right)$ remained approximately constant with increasing DC voltage until stick-slip initiated. Hysteresis initially decreased with AC voltage then leveled off to a small value at $V_{\text {rsm }} \sim 51 \mathrm{~V}$ where it remained until stick-slip initiated at higher voltages.

Similar changes in CA hysteresis have been observed by others under DC and AC polarization ( $\mathrm{Li}$ and Mugele 2008) and under DC polarization (Gupta et al. 2011). Li and Mugele reported CA hysteresis decreases with AC polarization and attributed the decreases to field-induced triple-line oscillations providing sufficient energy to overcome localized pinning. Their data cover EWOD polarization values, expressed in terms of electrowetting number, ${ }^{2}$ ranging from 0 to 0.2 (DC and AC-rms). They also mentioned "increased spikiness" of the CA at higher voltage levels consistent with the stick-slip behavior in this work, which occurs near EWOD saturation at an electrowetting number of $\sim 1.0$.

\footnotetext{
2 The electrowetting number is defined by: $E W=\left(\varepsilon_{0} \varepsilon_{d} / 2 \gamma t\right) \times V^{2}$.
}

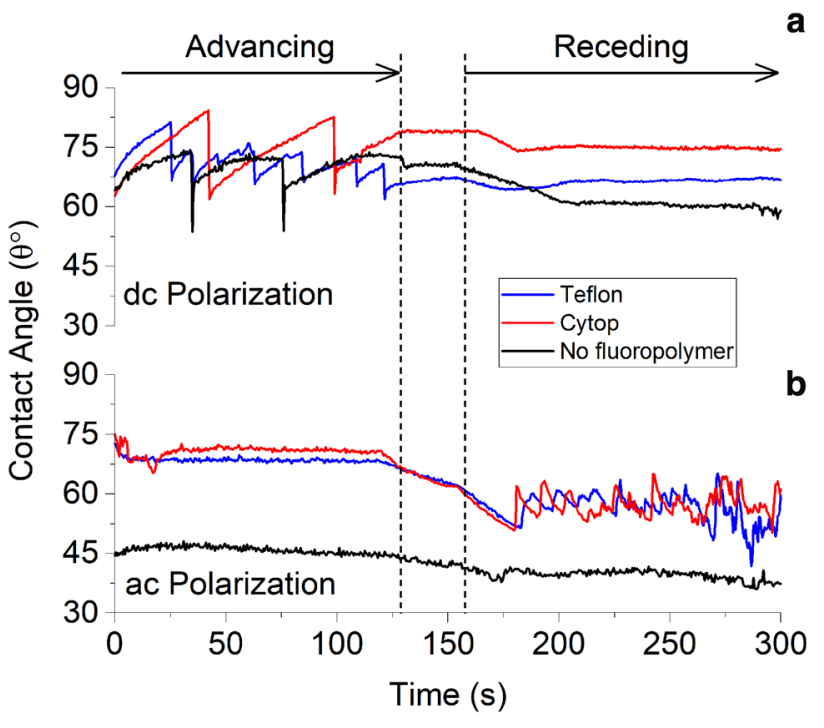

Fig. 3 Substrate coating effects on the advancing/receding CA for DC and AC polarization. $V_{\mathrm{dc}}=160 \mathrm{~V}(\mathbf{a}), V_{\mathrm{rsm}}=144 \mathrm{~V}(\mathbf{b})$. The vertical lines indicating the advancing and receding times are approximate

Figure 2 shows CA oscillations at the high DC and AC voltages along with the corresponding droplet base width (diameter). The CA gradually increases with DC polarization during advance as the droplet volume increases while the diameter remains constant due to triple-line pinning. An abrupt decrease in CA occurs when slip initiates, and this is accompanied by a rapid increase in diameter that overshoots slightly and then quickly equilibrates at a slightly smaller diameter. This same cycle then repeats. Similar behavior occurs with AC polarization during retreat with droplet volume decreases. The $\mathrm{AC}$ responses during retreat do not show stick-slip as distinctly as the DC data. Droplets often exhibited asymmetric distortions of the triple-line and movement relative to the dispensing needle (e.g., see Figure S2 in Online Resource 1) during stick-slip. The droplet-substrate interface zone was more asymmetrical in shape with $\mathrm{AC}$ polarization, and when it occurred, it produced more erratic diameter values and fewer plateaus (Fig. 2b). Figure S3 (in Online Resource 1) links to videos showing triple-line sticking at locations along the periphery, while the remaining droplet tries to push past (DC with increasing volume) or pull away (AC with decreasing volume).

Stick-slip behavior was observed at voltages as low as 50-V DC during CP-EWOD testing on conductive polyimide Kapton XC or RS substrates, which have a higher surface roughness than polished silicon and glass-ITO, but it was more distinct at higher positive and negative voltages (Merrill et al. 2018) (e.g., Figure S4; Online Resource 1). It persisted at lower voltages (even $0 \mathrm{~V}$ ) at substrate locations previously tested to $V_{\text {sat }}$ (DC or AC), but with smaller CA variations (history effect). It occurred on substrates with two 

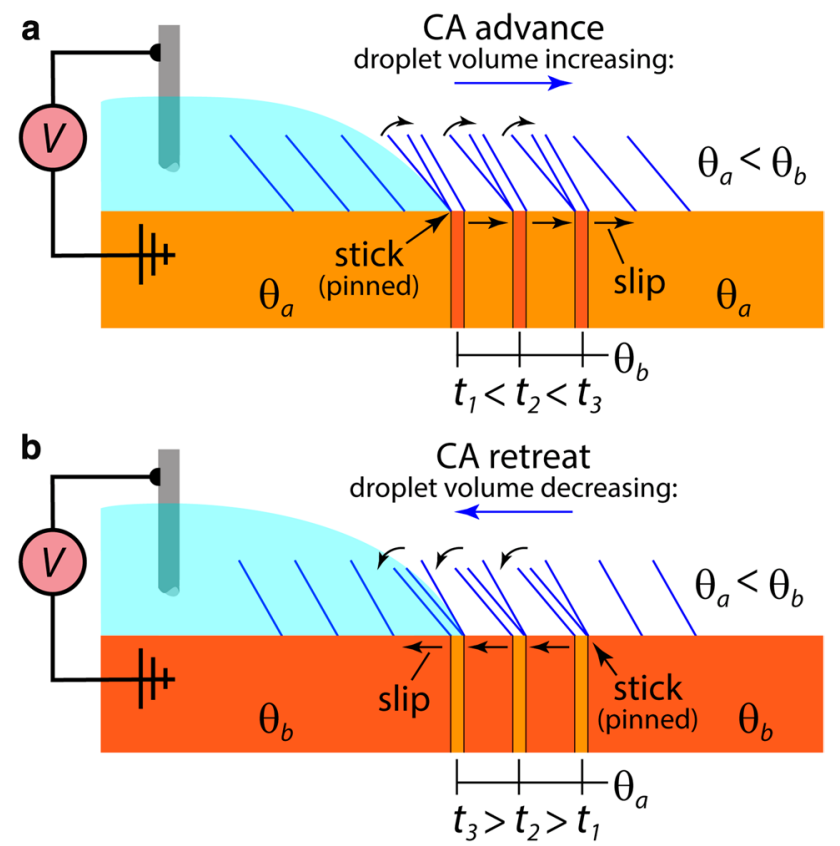

Fig. 4 Illustration showing how different surface wetting states that are created at each stick-slip cycle can produce triple-line stick-slip during: a advance but not retreat, and $\mathbf{b}$ retreat but not advance

different fluoropolymer coatings (Teflon and Cytop) but not on those coated with Parylene but no fluoropolymer under AC polarization (Fig. 3). Teflon and Cytop coatings are known to be susceptible to surface charging (Drygiannakis et al. 2009) by adsorbed $\mathrm{OH}^{-}$ions (Banpurkar et al. 2017; Zimmermann et al. 2001), but changes in $\mathrm{pH}$ and, thus, $\mathrm{OH}^{-}$concentration showed no significant effect (Figure S5; Online Resource 1). Stick-slip was greatly reduced at AC frequencies greater than $1 \mathrm{kHz}$ (Figure S6; Online Resource 1). On the polished Si and glass-ITO substrates, stick-slip was only observed at DC and AC voltages approaching saturation, which suggests a link between stick-slip and saturation.

Heterogeneous wetting surfaces can produce the types of stick-slip behavior observed in this work. The simple heuristic model provided in Fig. 4 illustrates how stick-slip can arise during triple-line advance or retreat on surfaces with two wetting zones ( $a$ and $\left.b, \theta_{a}<\theta_{b}\right)$ of different relative sizes. Surfaces with larger $\theta_{a}$ zones and smaller $\theta_{b}$ borders (e.g., Fig. 4a) will exhibit stick-slip in advance but not retreat. Conversely, surfaces with larger $\theta_{b}$ zones and smaller $\theta_{a}$ borders (e.g., Fig. $4 \mathrm{~b}$ ) will exhibit stick-slip in retreat but not advance. For sessile droplets, the wetting regions in Fig. 4 can be concentric rings around the droplet or small "island" zones randomly distributed on the surface. The small regions with different wetting behavior ( $\theta_{b}$ in Fig. $4 \mathrm{a}$ and $\theta_{a}$ in Fig. $4 \mathrm{~b}$ ) are formed during CP-EWOD at the droplet triple-line (liquid-solid interface), thus inducing stick. Immediately following slip, a new region will form at the triple-line with consequent sticking conditions. The multiple regions shown in Fig. 4 correspond to the triple-line location at successive times. The effect of triple-line pinning on CA variations during advance is explored further in the Online Resource 1 with matched experimental CA data and a model for the droplet diameter assuming spherical shapes (Figure S7a; Online Resource 1). MATLAB computations are used to demonstrate relative size effects for the two wetting states on the CA response during advance and retreat (Figure S7b-d; Online Resource 1).

Heterogeneous wetting on EWOD surfaces can arise with spatial variations in the dielectric coating thickness, sharp-edged surface roughness features, and charge trapping at saturation. In the trapping model proposed by Verheijen and Prins (1999), charged ionic species from the liquid are adsorbed on the dielectric surface. They may subsequently be absorbed and diffuse into the dielectric coating below the liquid-solid interface and extend a small distance in front of the triple-line (on the order of the dielectric coating thickness). The term "trapping" implies species immobilization due to stronger "bonding" affinity with the dielectric surface/ bulk than with the liquid phase. The presence of trapped charged species in the vicinity of the triple-line shields the electrostatic force (Maxwell stress) acting on the droplet liquid-air interface near the triple-line (Kang 2002; Hong et al. 2008). This leads to deviations from idealized Lippmann-Young behavior. A simple modification to the Lippmann-Young equation was proposed to account for charge trapping (Verheijen and Prins 1999):

$\cos \theta=\cos \theta_{0}+\frac{\varepsilon \varepsilon_{0}}{2 \gamma t}\left(V-V_{T}\right)^{2}$,

where $\theta$ is the CA at a given applied DC voltage, $\theta_{0}$ is the zero-voltage contact angle, $\varepsilon_{0}$ and $\varepsilon$ are the permittivity of vacuum and relative permittivity, respectively, $\gamma$ is surface tension, $t$ is dielectric coating thickness, $V$ is the applied potential, $V_{T}$ is the effective potential of the trapped charge, and $\left(V-V_{T}\right)$ is the equivalent EWOD voltage. The trapping of charged species with the same polarity as the applied voltage reduces the electrostatic "spreading" force producing an apparent reduced-wetting surface (i.e., larger CA). The trapped species distribution at the triple-line (surface/ bulk concentration versus location) is expected to primarily influence the magnitude of $V_{T}$.

Trapping under DC polarization can produce heterogeneous wetting conditions similar to that shown in Fig. 4a. Small $\theta_{b}$ zones with widths on the order of the dielectric thickness created by charge trapping at the onset of saturation would produce CA oscillations during triple-line advance. The absence of CA plateauing during 
advance (constant CA versus time) and little or no oscillation during droplet retreat is consistent with relatively small, closely spaced $\theta_{b}$ regions. In earlier work (Merrill et al. 2018), similar CA oscillation behavior with droplet expansion was shown under both positive and negative DC polarization.

Stick-slip during retreat under AC polarization is consistent with the heterogeneous wetting conditions shown in Fig. 4b: small $\theta_{a}$ zones with a reduced CA surrounded by larger $\theta_{b}$ regions (i.e., $\theta_{a}<\theta_{b}$ ). Small $\theta_{a}$ regions with lower apparent $\mathrm{CA}$ created by trapping under AC polarization would require the trapped species to produce an effective increase in the EWOD voltage, which is opposite to the effect of trapping under DC polarization. It can be explained by an analysis of the effects of charge trapping on the rsm and rms (DC-equivalent) voltages (see Appendix and Online Resource 1). Both of these voltage measures show an increase in magnitude when a single-charged species is trapped over the positive and negative phases of the voltage cycle (see Tables S2 and S3 in Online Resource 1). The resulting Lippmann-Young equation for each AC voltage representation, assuming single-species (polarity) trapping, are given by:

$$
\begin{aligned}
& \mathrm{rsm}: \cos \theta=\cos \theta_{0}+\frac{\varepsilon \varepsilon_{0}}{2 \gamma t}\left(\left(\frac{2 A}{\pi}\right)^{2}+V_{T}^{2}\right), \\
& \mathrm{rms:} \cos \theta=\cos \theta_{0}+\frac{\varepsilon \varepsilon_{0}}{2 \gamma t}\left(\frac{A^{2}}{2}+V_{T}^{2}\right),
\end{aligned}
$$

where $A$ is the $\mathrm{AC}$ voltage amplitude and the other variables are defined as for Eq. (1). A similar rms voltage with a positive offset was obtained by Bonfante et al. (Eq. 5, Bonfante et al. 2017) in modeling an oil droplet in an electrolyte with condensation of surface charge on the dielectric layer.

Single-species trapping under AC polarization may be possible under certain conditions as explained below. If the polarity of the trapped species follows the applied EWOD voltage polarity over each AC half-cycle, then the rsm effective voltage is reduced by the trapping voltage in the same manner as with DC trapping, while the rms effective voltage behaves in a more complex manner. Tables S1 through S3 in the Online Resource 1 provide expressions for three different DC-equivalent representations under four different possible trapping conditions.

Trapping and de-trapping of charged species from the surface and/or in the bulk of the dielectric coating(s) during EWOD polarization are physico-chemical processes with dynamics governed by the kinetics of multiple mechanistic steps. While the details are beyond the scope of this work, some of the relevant steps in trapping likely include: charged species transport through the bulk droplet liquid to the double-layer at the liquid-solid interface by electromigration, diffusion, and convection; adsorption on the dielectric surface; surface trapping and diffusion; absorption in the bulk dielectric; transport by electromigration and diffusion in the dielectric layer(s); and bulk trapping. De-trapping will involve similar steps but in the reverse order. The dynamics of trapping and de-trapping of charged species in EWOD is expected to reflect the kinetics of the slowest (rate-controlling) step(s) relevant to each individual charged species.

The polarity of the species involved in trapping/detrapping will follow the applied (needle) polarity under AC polarization at low frequencies. The stick-slip behavior observed at positive and negative DC saturation voltages is an example of this in the limit of low frequency. As the applied AC frequency increases, but below the transition to dielectric liquid behavior, the rate-controlling step(s) for trapping and de-trapping for each individual charged species should begin to manifest. The extent of charged-species trapping over each phase of the AC polarization cycle presumably depends in a complex manner on the rates of trapping and de-trapping of each individual charged species. It is hypothesized that as the AC frequency increases, a single-charged species with the fastest overall trapping kinetics and slowest overall de-trapping kinetics (each controlled by their slowest rate-controlling step) will dominate and affect the AC EWOD saturation response. The stick-slip behavior observed at AC saturation voltages in this work is consistent with this proposed working hypothesis.

The general model outlined above identifies plausible mechanistic limitations on trapping/de-trapping that could produce heterogeneous surface wetting conditions consistent with the observed triple-line stick-slip behaviors. It also highlights the need for and opportunities provided by future studies of trapping mechanisms using the CPEWOD approach. For example, experiments could be conducted with variations in the AC EWOD frequencies (e.g., 0-20kHz), AC waveforms (sawtooth, DC bias), and system temperature to help identify operative trapping mechanisms, discriminate between rate-controlling trapping and de-trapping steps, and quantify individual step kinetics and activation energies.

Kilaru et al. (2007) studied the effects of $\mathrm{BaTiO}_{3}$ nanoparticles added to a fluoropolymer dielectric top-coat on the EWOD and surface charge responses. The results are interesting and relevant to this work because of the strong charge trapping exhibited by the nanocomposite dielectric configurations. The results showed CA saturation occurred at -60 to $-70 \mathrm{~V}$ DC with $50-75 \%$ (vol) $\mathrm{BaTiO}_{3}$ content. A novel capacitance-voltage experimental setup was employed to measure surface charge versus AC sinusoidal voltage $( \pm 20$ and $\pm 80 V_{\text {peak }} ; 1 \mathrm{kHz}$ ) on the 0,50 and $97 \% \mathrm{BaTiO}_{3}$ layers (Fig. 4 in Kilaru et al. 2007). Their Fig. 4a shows a nonsymmetrical hysteresis loop (evidence of trapping) in the $\pm 80 \mathrm{~V} \mathrm{AC}$ polarization results for voltages $>50 V_{\text {peak }}$ in the pure fluoropolymer, but only in the positive phase, not the 
negative phase. This result is consistent with single-species trapping under AC polarization, and their fluoropolymer materials are the same as those used in this work. The results in their Fig. 4b, c for the 50 and $97 \%$ (vol.) $\mathrm{BaTiO}_{3}$ layers show symmetrical hysteresis loops over the positive and negative $\mathrm{AC}$ voltage phases, with both the \pm 20 and $\pm 80 V_{\text {peak }}$ polarization conditions. These results are consistent with fast trapping and de-trapping kinetics (relative to the $1-\mathrm{kHz}$ polarization signal) that enable trapping/de-trapping of both positive and negative species following the applied AC polarity.

\section{Summary and conclusions}

Use of the CP-EWOD experimental technique uncovered novel triple-line stick-slip behavior not previously observed in works using a constant-volume, potential-raster approach. Heterogeneous wetting zones created by charged species trapping at saturation were proposed to explain the observations. The DC trapping model of Verheijen and Prins (1999) was extended to include trapping under AC polarization, which required a more thorough examination of the concept of a DC-equivalent for AC voltages in EWOD. A newly defined root-square-mean (rsm) voltage was shown to be better than the more common root-mean-square (rms) and the absolute magnitude (am) voltage measures. It provided: (a) a better match between AC and DC/Lippmann-Young EWOD CA responses; (b) predicted AC and DC trapping effects consistent with all of the experimental stick-slip data; and (c) an average liquid-solid interface charge density value identical with the integral-averaged charge density calculated using the time-dependent AC voltage. The trapping models represented by Eqs. (1) and (2) can explain the creation of different heterogeneous wetting zones near the triple-line under DC and AC polarization that give rise to the observed differences in stick-slip behaviors between DC and AC EWOD.

The apparent increase in the EWOD saturation voltage under AC polarization reported in many previous studies might be due to the use of $V_{\text {rms }}$ as a DC-equivalent representation. In the absence of trapping, $V_{\mathrm{rsm}} \sim 0.9 V_{\mathrm{rms}}$; its use in this work led to better agreement between the DC and AC EWOD responses and saturation voltage magnitudes. The utility of $V_{\text {rsm }}$ for AC-EWOD studies and applications should be further explored and experimentally validated. Experiments to better understand the effects of size and distribution of possible heterogeneous wetting zones on possible triple-line pinning under DC and AC polarization are also needed. CP-EWOD experiments on substrates with a dense dielectric coating such as silicon oxide and vapor-deposited PTFE could be used to minimize coating imperfections and substrate roughness effects that might affect trapping and would help isolate the effects of DC and $\mathrm{AC}$ voltage amplitude and $\mathrm{AC}$ frequency.

Acknowledgements Support for this work by the Air Force Office of Scientific Research (MIPR F4FGA05239G001) is gratefully acknowledged.

Open Access This article is licensed under a Creative Commons Attribution 4.0 International License, which permits use, sharing, adaptation, distribution and reproduction in any medium or format, as long as you give appropriate credit to the original author(s) and the source, provide a link to the Creative Commons licence, and indicate if changes were made. The images or other third party material in this article are included in the article's Creative Commons licence, unless indicated otherwise in a credit line to the material. If material is not included in the article's Creative Commons licence and your intended use is not permitted by statutory regulation or exceeds the permitted use, you will need to obtain permission directly from the copyright holder. To view a copy of this licence, visit http://creativecommons.org/licenses/by/4.0/.

\section{Appendix}

The equation for calculating the root-square-mean voltage, $V_{\text {rsm }}$, is provided below. Let $V_{\text {ac }}=A \sin \omega t-V_{T}(t)$, where $A$ is the AC voltage amplitude and $V_{T}(t)$ is the effective trapping voltage. If $V_{T}(t)$ is assumed to be constant (but possibly different) over each AC half-cycle, then $V_{\text {rsm }}$ is given by:

$$
\begin{aligned}
V_{\mathrm{rsm}} & =\sqrt{\frac{1}{2}\left[\left(\frac{1}{T / 2} \int_{0}^{T / 2}\left(A \sin \omega t-V_{T}^{+}\right) \mathrm{d} t\right)^{2}+\left(\frac{1}{T / 2} \int_{T / 2}^{T}\left(A \sin \omega t-V_{T}^{-}\right) \mathrm{d} t\right)^{2}\right]} \\
& =\sqrt{\left(\frac{2 A}{\pi}\right)^{2}+\frac{\left(V_{T}^{+}\right)^{2}+\left(V_{T}^{-}\right)^{2}}{2}-\frac{2 A\left(V_{T}^{+}-V_{T}^{-}\right)}{\pi}} .
\end{aligned}
$$


The rsm voltage used in Eq. (2) is obtained by setting $V_{T}^{+}=V_{T}^{-}=V_{T}$ in Eq. (3). The root-mean-square voltage, $V_{\text {rms }}$, differs by squaring the voltage expression, $V_{a c}$, before integrating. Additional details and analysis can be found in the Online Resources 1.

\section{References}

Banpurkar AG, Sawane Y, Wadhai SM, Murade CU, Siretanu I, van den Ende D, Mugele F (2017) Spontaneous electrification of fluoropolymer-water interfaces probed by electrowetting. Faraday Discuss 199:29-47. https://doi.org/10.1039/c6fd00245e

Bonfante G, Roux-Marchand T, Audry-Deschamps MC, Renaud L, Kleimann P, Brioude A, Maillard M (2017) Polarization mechanisms of dielectric materials at a binary liquid interface: impacts on electrowetting actuation. Phys Chem Chem Phys 19:30139-30146. https://doi.org/10.1039/c7cp06052a

Charipar KM, Charipar NA, Bellemare JV, Peak JE, Pique A (2015) Electrowetting displays utilizing bistable, multi-color pixels via laser processing. J Display Technol 11:175-182. https:// doi.org/10.1109/Jdt.2014.2364189

David R, Neumann AW (2013) Contact angle hysteresis on randomly rough surfaces: a computational study. Langmuir 29:45514558. https://doi.org/10.1021/la400294t

David R, Neumann W (2010) Computation of contact lines on randomly heterogeneous surfaces. Langmuir 26:13256-13262. https://doi.org/10.1021/la102605g

Diebold AV, Watson AM, Holcomb S, Tabor C, Mast D, Dickey MD, Heikenfeld J (2017) Electrowetting-actuated liquid metal for RF applications. J Micromech Microeng. https://doi. org/10.1088/1361-6439/aa556a

Drygiannakis AI, Papathanasiou AG, Boudouvis AG (2009) On the connection between dielectric breakdown strength, trapping of charge, and contact angle saturation in electrowetting. Langmuir 25:147-152. https://doi.org/10.1021/La802551j

Fair RB (2007) Digital microfluidics: Is a true lab-on-a-chip possible? Microfluid Nanofluid 3:245-281. https://doi.org/10.1007/ s10404-007-0161-8

Gupta R, Sheth DM, Boone TK, Sevilla AB, Frechette J (2011) Impact of pinning of the triple contact line on electrowetting performance. Langmuir 27:14923-14929. https://doi. org/10.1021/la203320g

Hong JS, Ko SH, Kang KH, Kang IS (2008) A numerical investigation on AC electrowetting of a droplet. Microfluid Nanofluid 5:263-271. https://doi.org/10.1007/s10404-007-0246-4

Huh C, Mason SG (1977) Effects of surface-roughness on wetting (theoretical). J Colloid Interface Sci 60:11-38. https://doi. org/10.1016/0021-9797(77)90251-X

Johnson RE, Dettre RH (1964) Contact angle hysteresis. 3. Study of an idealized heterogeneous surface. J Phys Chem 68:1744. https ://doi.org/10.1021/j100789a012

Kang KH (2002) How electrostatic fields change contact angle in electrowetting. Langmuir 18:10318-10322. https://doi. org/10.1021/La0263615

Kedzierski J, Meng K, Thorsen T, Cabrera R, Berry S (2016) Microhydraulic electrowetting actuators. J Microelectromech Syst 25:394-400. https://doi.org/10.1109/Jmems.2016.2521439

Kilaru MK, Heikenfeld J, Lin G, Mark JE (2007) Strong charge trapping and bistable electrowetting on nanocomposite fluoropolymer: BaTiO3 dielectrics. Appl Phys Lett 90:212906. https://doi. org/10.1063/1.2743388
Krupenkin T, Taylor JA (2011) Reverse electrowetting as a new approach to high-power energy harvesting. Nat Commun. https ://doi.org/10.1038/ncomms1454

Kwok DY, Lam CNC, Li A, Leung A, Wu R, Mok E, Neumann AW (1998) Measuring and interpreting contact angles: a complex issue. Colloid Surface A 142:219-235. https://doi.org/10.1016/ S0927-7757(98)00354-9

Leopoldes J, Bucknall DG (2005) Droplet spreading on microstriped surfaces. J Phys Chem B 109:8973-8977. https://doi.org/10.1021/ jp0508094

Li D, Cheng P, Neumann AW (1992) Contact angle measurement by axisymmetric drop shape analysis (ADSA). Adv Coll Interface Sci 39:347-382. https://doi.org/10.1016/0001-8686(92)80065-6

Li F, Mugele F (2008) How to make sticky surfaces slippery: contact angle hysteresis in electrowetting with alternating voltage. Appl Phys Lett. https://doi.org/10.1063/1.2945803

Li Q, Zhou P, Yan HJ (2016) Pinning-depinning mechanism of the contact line during evaporation on chemically patterned surfaces: a Lattice Boltzmann study. Langmuir 32:9389-9396. https://doi. org/10.1021/acs.langmuir.6b01490

Long J, Hyder MN, Huang RYM, Chen P (2005) Thermodynamic modeling of contact angles on rough, heterogeneous surfaces. Adv Coll Interface Sci 118:173-190. https://doi.org/10.1016/j. cis.2005.07.004

Merrill MH, Reid RC, Gogotsi N, Thomas JP (2018) Electrowetting on polyimide and silicon substrates with high hysteresis. Microsyst Technol. https://doi.org/10.1007/s00542-018-3896-0

Merrill MH, Thomas JP, Auyeung RCY, Piqué A (2014) Electrowetting actuation methods for surface morphology control of multifunctional composites. In: Paper presented at the American Society for Composites-29th Technical Conference, La Jolla, CA

Mishra K et al (2014) Optofluidic lens with tunable focal length and asphericity. Sci Rep. https://doi.org/10.1038/srep06378

Mishra K, van den Ende D, Mugele F (2016) Recent developments in optofluidic lens technology. Micromachines. https://doi. org/10.3390/mi7060102

Mugele F, Baret JC (2005) Electrowetting: from basics to applications. J Phys Condens Matter 17:R705-R774. https://doi. org/10.1088/0953-8984/17/28/R01

Nelson WC, Sen P, Kim CJ (2011) Dynamic contact angles and hysteresis under electrowetting-on-dielectric. Langmuir 27:1031910326. https://doi.org/10.1021/la2018083

Oliver JF, Huh C, Mason SG (1980) Experimental-study of some effects of solid-surface roughness on wetting. Colloid Surface 1:79-104. https://doi.org/10.1016/0166-6622(80)80039-4

Priest C, Sedev R, Ralston J (2007) Asymmetric wetting hysteresis on chemical defects. Phys Rev Lett. https://doi.org/10.1103/PhysR evLett.99.026103

Priest C, Sedev R, Ralston J (2013) A quantitative experimental study of wetting hysteresis on discrete and continuous chemical heterogeneities. Colloid Polym Sci 291:271-277. https://doi. org/10.1007/s00396-012-2758-Z

Prins MWJ, Welters WJJ, Weekamp JW (2001) Fluid control in multichannel structures by electrocapillary pressure. Science 291:277280. https://doi.org/10.1126/science.291.5502.277

Raj R, Enright R, Zhu YY, Adera S, Wang EN (2012) Unified model for contact angle hysteresis on heterogeneous and superhydrophobic surfaces. Langmuir 28:15777-15788. https://doi.org/10.1021/ la303070s

Reid RC, Thomas JP, Merrill MH (2017) Advances in poro-vascular composites: toward non-mechanical surface roughness control. In: Paper presented at the 21st International Conference on Composite Materials (ICCM-21), Xi' an, China 
Samiei E, Tabrizian M, Hoorfar M (2016) A review of digital microfluidics as portable platforms for lab-on a-chip applications. Lab Chip 16:2376-2396. https://doi.org/10.1039/c6lc00387g

Sen P, Kim CJ (2009) Capillary spreading dynamics of electrowetted sessile droplets in air. Langmuir 25:4302-4305. https://doi. org/10.1021/la900077u

Shanahan MER (1991) A simple analysis of local wetting hysteresis on a Wilhelmy plate. Surf Interface Anal 17:489-495. https://doi. org/10.1002/sia.740170713

Tavana $\mathrm{H}$ et al (2006) Stick-slip of the three-phase line in measurements of dynamic contact angles. Langmuir 22:628-636. https:// doi.org/10.1021/la051715o

Verheijen HJJ, Prins MWJ (1999) Reversible electrowetting and trapping of charge: model and experiments. Langmuir 15:6616-6620. https://doi.org/10.1021/La990548n

Wijewardhana KR, Shen TZ, Song JK (2017) Energy harvesting using air bubbles on hydrophobic surfaces containing embedded charges. Appl Energ 206:432-438. https://doi.org/10.1016/j.apene rgy.2017.08.211
You H, Steckl AJ (2010) Three-color electrowetting display device for electronic paper. Appl Phys Lett. https://doi.org/10.1063/1.34649 63

Zimmermann R, Dukhin S, Werner C (2001) Electrokinetic measurements reveal interfacial charge at polymer films caused by simple electrolyte ions. J Phys Chem B 105:8544-8549. https://doi. org/10.1021/jp004051u

Zuo BA, Zheng FF, Zhao YR, Chen T, Yan ZH, Ni HG, Wang XP (2012) Stick-slip phenomenon in measurements of dynamic contact angles and surface viscoelasticity of poly(styrene-b-isopreneb-styrene) Triblock copolymers. Langmuir 28:4283-4292. https ://doi.org/10.1021/la300119n

Publisher's Note Springer Nature remains neutral with regard to jurisdictional claims in published maps and institutional affiliations. 